\title{
STRONG ANISOTROPIC FLOW IN A FINELY LAYERED ASTHENOSPHERE
}

\author{
Satoru Honda
}

Seismological Laboratory, California Institute of Technology, Pasadena, CA 91125

\begin{abstract}
We derive the long wave length equivalents of the constitutive relations for finely stratified fluids as suggested by Saito and Abe [1984]. The results show the transversely isotropic relations characterized by two effective viscosities. One is associated with shear stress applied on the plane parallel to the layering and the other is with normal stress applied perpendicular to the layering. If thin soft layers exist in the low velocity zone, there may be a significant difference in these two viscosities. A simple calculation simulating mantle flow with large apparent anisotropy shows the possible existence of convection having large aspect ratio.
\end{abstract}

\section{Introduction}

It is often believed that the low velocity zone is a partially molten layer le.g., Anderson and Sammis, 1970]. Post glacial rebound studies conclude the existence of a low viscosity zone around the seismic low velocity zone, suggesting the existence of soft layers [e.g., Cathles, 1975]. For these reasons, previous investigators assumed the presence of a low viscosity zone around $100 \mathrm{~km}$ depth [e.g., Houston and De Bremaecker, 1975]. If the melts are distributed uniformly, such a layer may be regarded as an isotropic fluid. However, as pointed out by Saito and Abe [1984] (here after referred to as SA), if the low viscosity zone consists of a laminated medium of horizontal hard and soft layers, its treatment as an isotropic fluid with low viscosity may break down. Such a laminated mantle has been proposed by several authors to explain the discrepancies of Love and Rayleigh waves dispersions [e.g., Aki, 1968; Takeuchi et al., 1968]. If a horizontal shear stress is applied, the deformation will be controlled by the soft layers. On the contrary, when a vertical normal stress is applied, the deformation will be controlled by the hard layers. Such a body may be treated as an anisotropic fluid for scale length much larger than the thickness of layering. This kind of apparent anisotropy is well known in the field of seismology as "transverse isotropy" [Backus, 1962]. Backus [1962] only considered the case of elastic solids. The main purpose of this paper is to derive the long wave length equivalents of constitutive relations for finely stratified fluids by following Backus [1962] and show the potential importance of such an apparent anisotropy. SA quoted Backus [1962] in their application of their theory of anisotropic fluids. However, there is an error which we will point out later. We also calculate simple 2-D flow models to demonstrate the importance of apparent anisotropy. Christensen [1986] also discussed these problems mainly based on numerical simulations, though he did

Copyright 1986 by the American Geophysical Union.

Paper number 6L7044.

0094-8276/86/006L-7044\$03.00 not consider the layering of partial melt zone which shows more drastic effects.

\section{Backus' Theory for Incompressible Fluid}

The constitutive relations for an incompressible fluid may be written as,

$$
\tau_{\mathrm{ij}}=-\mathrm{p} \delta_{\mathrm{ij}}+\eta\left(\partial \mathrm{u}_{\mathrm{i}} / \partial \mathrm{x}_{\mathrm{j}}+\partial \mathrm{u}_{\mathrm{j}} / \partial \mathrm{x}_{\mathrm{i}}\right)
$$

with

$$
\partial \mathrm{u}_{\mathrm{i}} / \partial \mathrm{x}_{\mathrm{i}}=0
$$

where $\tau_{\mathrm{ij}}(\mathrm{i}, \mathrm{j}=1,2,3)$ is the stress tensor, $p$ is the pressure, $u_{i}$ is the velocity, $x_{i}$ is the spatial coordinates and $\eta$ is the viscosity. We assume that the direction of stratification is $x_{3}$. Consider a scale length $\lambda^{\prime}$, which is much longer than the characteristic length of layering, though it is much shorter than the characteristic length of flow $\lambda$ under consideration. We introduce the following averaging,

$$
\left\langle\mathrm{f}\left(\mathrm{x}_{3}\right)\right\rangle=\int_{-\infty}^{+\infty} \mathrm{w}\left(\varsigma-\mathrm{x}_{3}\right) \mathrm{f}(\varsigma) \mathrm{d} \varsigma
$$

where the weighting function $w\left(x_{3}\right)$ is selected so as to average $\mathrm{f}\left(\mathrm{x}_{3}\right)$ roughly over a distance $\lambda^{\prime}$ around $x_{3}$ (see Backus [1962] for detail). As suggested by Backus [1962], if the fluid is stratified with varying viscosities, the quantities $\tau_{13}, \tau_{23}, \tau_{33}, \partial \mathrm{u}_{\mathrm{i}} / \partial \mathrm{x}_{1}$ and $\partial \mathrm{u}_{\mathrm{i}} / \partial \mathrm{x}_{2}$ may vary slowly with $x_{3}$ (named as SVQ). Other quantities $\tau_{11}, \tau_{12}, \tau_{22}$ and $\partial \mathrm{u}_{\mathrm{i}} / \partial \mathrm{x}_{3}$ may vary rapidly with $x_{3}$ (named as RVQ). The important point of Backus' theory is the assumption that

$$
<(\mathrm{RVQ})(\mathrm{SVQ})>=<(\mathrm{RVQ})><(\mathrm{SVQ})>
$$

and the equation,

$$
<\partial \mathrm{f}\left(\mathrm{x}_{1}, \mathrm{x}_{2}, \mathrm{x}_{3}\right) / \partial \mathrm{x}_{\mathrm{i}}>=\partial<\mathrm{f}\left(\mathrm{x}_{1}, \mathrm{x}_{2}, \mathrm{x}_{3}\right)>/ \partial \mathrm{x}_{\mathrm{i}}
$$

( $i=1,2,3$ : The third equation $i=3$ is valid, since we assume $\mathrm{w}(+\infty)=\mathrm{w}(-\infty)=0)$ To use these relations, we modify (1) to,

$$
\begin{gathered}
\tau_{11}=-\mathrm{p}+2 \eta \partial \mathrm{u}_{1} / \partial \mathrm{x}_{1} \\
\tau_{22}=-\mathrm{p}+2 \eta \partial \mathrm{u}_{2} / \partial \mathrm{x}_{2} \\
\tau_{33}=-\mathrm{p}-2 \eta\left(\partial \mathrm{u}_{1} / \partial \mathrm{x}_{1}+\partial \mathrm{u}_{2} / \partial \mathrm{x}_{2}\right) \\
\left(\text { Note } \partial \mathrm{u}_{\mathrm{i}} / \partial \mathrm{x}_{\mathrm{i}}=0\right) \\
\tau_{12}=\eta\left(\partial \mathrm{u}_{2} / \partial \mathrm{x}_{1}+\partial \mathrm{u}_{1} / \partial \mathrm{x}_{2}\right) \\
\partial \mathrm{u}_{2} / \partial \mathrm{x}_{3}=(1 / \eta) \tau_{23}-\partial \mathrm{u}_{3} / \partial \mathrm{x}_{2} \\
\partial \mathrm{u}_{1} / \partial \mathrm{x}_{3}=(1 / \eta) \tau_{31}-\partial \mathrm{u}_{3} / \partial \mathrm{x}_{1}
\end{gathered}
$$

(Note that these equations are of the form RVQ times SVQ.) We average the equations (6) and reorganize them as follows,

$$
\begin{aligned}
& <\tau_{11}>=-<\mathrm{p}>+2<\eta>\partial<\mathrm{u}_{1}>/ \partial \mathrm{x}_{1} \\
& <\tau_{22}>=-<\mathrm{p}>+2<\eta>\partial<\mathrm{u}_{2}>/ \partial \mathrm{x}_{2} \\
& <\tau_{33}>=-<\mathrm{p}>+2<\eta>\partial<\mathrm{u}_{3}>/ \partial \mathrm{x}_{3}
\end{aligned}
$$




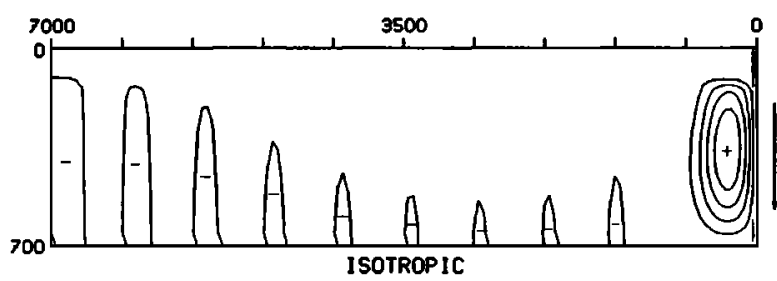

Fig. 1. Stream lines for isotropic low viscosity zone model. Only $0 \leq \mathrm{x}_{1} \leq l / 4$ is shown. The width of tick marks is $700 \mathrm{~km}$. Contour interval is 0.2 times absolute $\max$ of stream functions. The apparent periodicity of contour lines $(=0)$ is due to the truncation of Fourier series. Hatched area shows the position of sinking slab. Absolute $\max =3.85 \mathrm{~cm}^{2} / \mathrm{sec}$.

$$
\begin{aligned}
& \left(\text { Note } \partial<\mathrm{u}_{\mathrm{i}}>/ \partial \mathrm{x}_{\mathrm{i}}=0\right) \\
& <\tau_{12}>=\left\langle\eta>\left(\partial<\mathrm{u}_{2}>/ \partial \mathrm{x}_{1}+\partial<\mathrm{u}_{1}>/ \partial \mathrm{x}_{2}\right)\right. \\
& <\tau_{23}>=<\eta^{-1}>^{-1}\left(\partial<\mathrm{u}_{2}>/ \partial \mathrm{x}_{3}+\partial<\mathrm{u}_{3}>/ \partial \mathrm{x}_{2}\right) \\
& <\tau_{31}>=<\eta^{-1}>^{-1}\left(\partial<\mathrm{u}_{1}>/ \partial \mathrm{x}_{3}+\partial<\mathrm{u}_{3}>/ \partial \mathrm{x}_{1}\right)
\end{aligned}
$$

Backus [1962] showed that, for the long wave length, the velocity field, $u_{i}$ could be approximately calculated by using equations (7) as the constitutive relations. It is clear that equations (7) are the special case of transversely isotropic medium characterized by two viscosities $\langle\eta\rangle$ and $\left\langle\eta^{-1}\right\rangle^{-1}$ (see $\mathrm{SA}$ and Love [1926]). The former viscosity is associated with the normal stress applied to the plane of layering, and the second is with the shear stress applied parallel to the plane of layering.

Following SA, we introduce two parameters as follows,

$$
\begin{aligned}
\mathrm{L} & =\left\langle\eta^{-1}\right\rangle^{-1} \\
\delta & =\langle\eta\rangle / \mathrm{L}=\langle\eta\rangle\left\langle\eta^{-1}\right\rangle
\end{aligned}
$$

We note that $\delta$ here is different from that of SA. This difference arises, because SA did not consider the pressure terms $p$ and the incompressibility condition (2). For an isotropic fluid, $\mathrm{L}=\eta$ and $\delta=1$. If the viscosity ratio of hard $\left(\eta_{1}\right)$ and soft layers $\left(\eta_{2}\right)$ are large $\left(\eta_{1}\right.$ $\left.>>\eta_{2}\right), \delta$ may take very large values. Assume $c$ is the volume fraction of soft layers and $c<<1$. Then $\langle\eta\rangle$ is of the order of $\eta_{1}$ and $\left\langle\eta^{-1}\right\rangle$ is $c / \eta_{2}$. Combining these, $\delta \approx c \eta_{1} / \eta_{2}$. If we assume that $c \approx 10^{-3}$, $\eta_{1} \approx 10^{20}$ poise and $\eta_{2} \approx 10^{5}$ poise, then we obtain $\delta \approx$ $10^{12}$. SA suggested $\delta \approx 10^{6}$ (Even though the equation of $\delta$ is not valid, their estimate is still correct. This is because $\eta_{1} / \eta_{2}$ is much larger than the order of one.).

\section{Summary of Saito and Abe [1984]}

SA calculated the spectra of viscous relaxations and the marginal stability curves of Rayleigh - Benard convection in a transverse isotropic fluid. Material parameters are assumed to be constant throughout the fluid. They found that the viscous relaxation time is almost constant over a wide range of wave lengths for large $\delta$. Their results are $(\delta>>1)$,

$$
\rho \mathrm{g} \tau \mathrm{H} / 2 \mathrm{~L} \approx 2 \delta \quad(1 / 2 \sqrt{\delta} \quad \ll \mathrm{kH} \quad<2 \sqrt{\delta})
$$

where $\rho$ is the density, $g$ is the gravity, $\tau$ is the relaxation time, $k$ is the wave number, and $H$ is the layer thickness. This equation suggests that, if we neglect a strong apparent anisotropy due to layering in the mantle, what we obtain from the post glacial studies may be the viscosity value of $\delta \mathrm{L}=\langle\eta\rangle$, instead of $L$ value, as usually believed [SA]. Similar phenomena occur in the case of Rayleigh - Benard convection problems. The marginal stability curves of Rayleigh number versus wave length have a flat bottom over a wide range of wave length. This implies possibility of multi-scale convection including the convection with large aspect ratio ( $=$ (horizontal scale) / (vertical scale)). Their results show that the critical Rayleigh number, $\mathrm{Ra}_{\mathrm{c}}$, is,

$$
\mathrm{Ra}_{\mathrm{c}} \approx 4 \pi^{4} \delta \quad(\pi / 2 \sqrt{\delta}<\mathrm{kH} \ll \pi: \text { for large } \delta)
$$

Here $\mathrm{Ra}$ is the Rayleigh number defined as,

$$
\mathrm{Ra} \equiv \rho \mathrm{g} \alpha \beta \mathrm{H}^{4} / \kappa \mathrm{L}
$$

where $\alpha$ is the volume thermal expansion coefficient, $\beta$ is the temperature gradient and $\kappa$ is the thermal diffusivity of fluids. If we define $\delta \mathrm{L}$ as an effective viscosity, equation (10) gives the same result as that of isotropic fluid [Chandrasekhar, 1961]. Richter and Daly [1978] also discussed the consequences of anisotropic viscosity, but their derivation of equation of motion is empirical and they did not consider physical cause of such anisotropy in detail.

\section{2-D Problem With Propagator Matrix}

Consider 2-D problems (i.e., $\partial / \partial \mathrm{x}_{2}=0$ ). We assume that $u_{3}, u_{1}, \tau_{33}, \tau_{11}, \tau_{13}, p$ can be expressed by the Fourier series as

$$
\left(\begin{array}{c}
\mathrm{u}_{3}\left(\mathrm{x}_{1}, \mathrm{x}_{3}\right) \\
\mathrm{u}_{1}\left(\mathrm{x}_{1}, \mathrm{x}_{3}\right) \\
\tau_{33}\left(\mathrm{x}_{1}, \mathrm{x}_{3}\right) \\
\tau_{11}\left(\mathrm{x}_{1}, \mathrm{x}_{3}\right) \\
\tau_{13}\left(\mathrm{x}_{1}, \mathrm{x}_{3}\right) \\
\mathrm{p}\left(\mathrm{x}_{1}, \mathrm{x}_{3}\right)
\end{array}\right)=\sum_{\mathrm{n}=1}^{\infty}\left(\begin{array}{c}
\mathrm{u}_{3}^{\mathrm{n}}\left(\mathrm{x}_{3}\right) \cos \left(\mathrm{k}_{\mathrm{n}} \mathrm{x}_{1}\right) \\
\mathrm{u}_{1}^{\mathrm{n}}\left(\mathrm{x}_{3}\right) \sin \left(\mathrm{k}_{\mathrm{n}} \mathrm{x}_{1}\right) \\
\tau_{33}^{\mathrm{n}}\left(\mathrm{x}_{3}\right) \cos \left(\mathrm{k}_{\mathrm{n}} \mathrm{x}_{1}\right) \\
\tau_{11}^{\mathrm{n}}\left(\mathrm{x}_{3}\right) \cos \left(\mathrm{k}_{\mathrm{n}} \mathrm{x}_{1}\right) \\
\tau_{13}^{\mathrm{n}}\left(\mathrm{x}_{3}\right) \sin \left(\mathrm{k}_{\mathrm{n}} \mathrm{x}_{1)}\right. \\
\mathrm{p}^{\mathrm{n}}\left(\mathrm{x}_{3}\right) \cos \left(\mathrm{k}_{\mathrm{n}} \mathrm{x}_{1}\right)
\end{array}\right)
$$

where $k_{n}=2 \pi n / l . ~ l$ is the period for the horizontal direction. Combining the equations (12) with the equations of motion (neglecting the inertia term) and continuity, we obtain the following equations [Hager and O'Connel, 1981],

$$
\mathrm{Du}=\mathbf{A} \mathbf{u}
$$

where

$$
\begin{aligned}
& \mathbf{A}=\left(\begin{array}{cccc}
0 & -\mathrm{k} & 0 & 0 \\
\mathrm{k} & 0 & 0 & 2 \mathrm{k} \eta^{-1} \\
0 & 0 & 0 & -\mathrm{k} \\
0 & 2 \eta \mathrm{k} & \mathrm{k} & 0
\end{array}\right) \\
& \mathbf{u}=\left[\begin{array}{llll}
\mathbf{u}_{3} & \mathrm{u}_{1} & \tau_{11} / 2 \mathrm{k} & \tau_{13} / 2 \mathrm{k}
\end{array}\right]^{\mathrm{T}} \\
& \mathrm{D}=\mathrm{d} / \mathrm{dx}_{3}
\end{aligned}
$$

We dropped the suffix $n$ for simplicity. Equation (13) has the solution (now, we replace $x_{3}$ with $z$ ),

$$
\mathbf{u}(\mathrm{z})=\mathrm{e}^{\mathbf{A}\left(\mathbf{z}-\mathrm{z}_{0}\right)} \mathbf{u}\left(\mathrm{z}_{0}\right)
$$

If we assume that the width of the layering is much shorter than the wave length under consideration, that is, $\mathrm{k}\left(\mathrm{z}-\mathrm{z}_{0}\right)<<1$, we may expand $\mathrm{e}^{\mathrm{A}\left(\mathrm{z}-\mathrm{z}_{0}\right)}$,

$$
\mathrm{e}^{\mathbf{A}\left(\mathbf{z}-\mathbf{z}_{0}\right)} \approx \mathbf{I}+\mathbf{A}\left(\mathbf{z}-\mathbf{z}_{0}\right)
$$




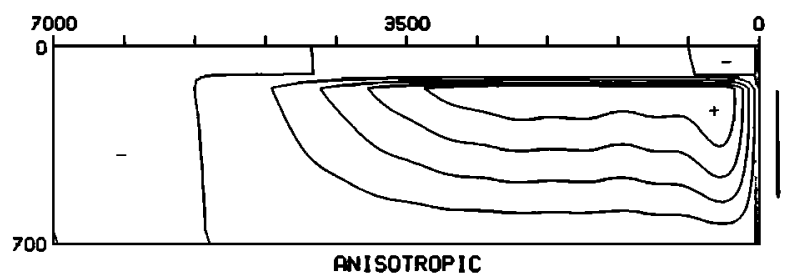

Fig. 2. Stream lines for anisotropic low viscosity zone model. See captions of Figure 1 for detail. Absolute $\max =13.8 \mathrm{~cm}^{2} / \mathrm{sec}$.

We neglected terms with the order higher than $O((\mathrm{k}(\mathrm{z}$ $\left.\left.\left.-z_{0}\right)\right)^{2}\right)$. We assume that there are many thin layers, with arbitrary constant viscosity, between $z_{0}$ and $z_{n}$, Then,

$$
u\left(z_{n}\right)=e^{A_{n}\left(z_{n}-z_{n-1}\right)} e^{A_{n-1}\left(z_{n-1}-z_{n-2}\right)} \ldots . . e^{A_{1}\left(z_{1}-z_{0}\right)} u\left(z_{0}\right)
$$

where the suffix $i$ attached to $\mathbf{A}$ signifies each layer which lies between $z_{\mathrm{i}}$ and $z_{\mathrm{i}-1}$. Considering (16), we may obtain,

$$
\mathrm{u}\left(\mathrm{z}_{\mathrm{n}}\right) \approx \mathrm{I}+\sum_{\mathrm{k}=1}^{\mathrm{n}} \mathbf{A}_{\mathrm{k}}\left(\mathrm{z}_{\mathrm{k}}-\mathrm{z}_{\mathrm{k}-1}\right) \equiv \mathbf{I}+\overline{\mathbf{A}}\left(\mathrm{z}_{\mathrm{n}}-\mathrm{z}_{0}\right)
$$

where $\overline{\mathbf{A}}$ is

$$
\begin{gathered}
\bar{A} \equiv\left(\begin{array}{cccc}
0 & -\mathrm{k} & 0 & 0 \\
\mathrm{k} & 0 & 0 & 2 \mathrm{k}<\eta^{-1}> \\
0 & 0 & 0 & -\mathrm{k} \\
0 & 2<\eta>\mathrm{k} & \mathrm{k} & 0
\end{array}\right)=\left(\begin{array}{cccc}
0 & -\mathrm{k} & 0 & 0 \\
\mathrm{k} & 0 & 0 & 2 \mathrm{~kL}^{-1} \\
0 & 0 & 0 & -\mathrm{k} \\
0 & 2 \delta \mathrm{Lk} & \mathrm{k} & 0
\end{array}\right) \\
<\mathrm{f}>=\int_{\mathrm{z}_{0}}^{\mathrm{z}_{\mathrm{n}}} \mathrm{f}(\mathrm{z}) \mathrm{dz} /\left(\mathrm{z}_{\mathrm{n}}-\mathrm{z}_{0}\right)
\end{gathered}
$$

Again, we neglected terms with the order higher than $O\left(\left(\mathrm{k}\left(\mathrm{z}-\mathrm{z}_{0}\right)\right)^{2}\right)$. This equation implies that, a fluid layer which includes many thin layers with arbitrary constant viscosity may be regarded as a transversely isotropic fluid characterized by two viscosities, $L$ and $\delta L$. This is the same conclusion discussed in the previous section.

\section{Numerical Examples}

We calculated the flow model following Hager and O'Connel [1981] to show the importance of the apparent anisotropy on the mantle convection. We consider 2-D problems in which the periodicity (with the period $l=28000 \mathrm{~km}$ ) in the horizontal direction is assumed as in the previous section. The depth of convection layer, bounded by the free top and bottom surfaces, is $700 \mathrm{~km}$. $x_{3}$ is assumed to be vertical, i.e. horizontal layering. The slab, with density contrast of $0.066 \mathrm{~g} / \mathrm{cm}^{3}$, subducts vertically at $l / 4=7000 \mathrm{~km}$. The thickness of the slab is $100 \mathrm{~km}$ and the penetration depth is $700 \mathrm{~km}$. Viscosity structure is similar to the one proposed by Cathles [1975]. A $10^{23}$ poise lithosphere of thickness $100 \mathrm{~km}$ overlies a $10^{22}$ poise mantle. We assume a $50 \mathrm{~km}$ thick low viscosity zone just beneath the lithosphere, with and without the apparent anisotropy. The flow (neglecting the inertia term) induced by this density contrast may be calculated by the propagator matrix technique described in the previous section. We consider two cases. First, we assume that the low viscosity zone is an isotropic fluid (i.e., $\delta=1$ ) of viscosity $10^{20}$ poise. The result is shown in Figure 1. The summation of Fourier series was done up to $n=40$. Basically, we obtained a cell of aspect ratio one. This has been one of the arguments in support of the whole mantle convection hypothesis. In the second case, we included the apparent anisotropic effect by changing from $\delta=1$ to $\delta=400$. However, we fixed the product as $\delta \mathrm{L}=10^{20}$ poise. This is because, as suggested by SA, the viscosity obtained by the post glacial uplift studies may be the product $\delta \mathrm{L}$. The flow field for the second case (Figure 2) changed very drastically, a cell of horizontal length scale about seven to eight times the depth appears. Also, we found that the amplitude becomes larger than the isotropic case. These results suggest the importance of the apparent anisotropy associated with the stratification of low viscosity zone.

\section{Discussion}

The key point of our discussions depends on the melt geometry of the low viscosity zone. Although Waff [1980] discussed the possibility of horizontal layering of melts, it is still controversial. There are several methods to determine the possible existence of melt zone layering. Seismic studies require the transversely isotropic upper mantle which may be produced by horizontal layering [Anderson and Dziewonski, 1982]. Probably, the most direct method of detecting the horizontal layering is to find the anisotropy in the electrical conductivity, though it is not perfect.

Another type of layering was suggested by Christensen [1986] such as the one produced by the chemical differences between the mantle and subducted crust. We assume that the anisotropy is fixed in the spatial coordinates. This assumption may be valid, if the temperature within the mantle is in a quasi steady state. Christensen [1986] made a fully dynamic calculation so that the anisotropy is carried by the convective flow. Although, he assumed a weak anisotropy, he found the interesting result that the center of the convection cell is almost fixed in space. Clearly, the inclusion of the apparent anisotropy produces many interesting phenomena which deserve further investigation. We did not carry out the test of stability of flows. Such a dynamical calculation may be required in the future studies.

Acknowledgements. This work was stimulated by the suggestions of Saito and Abe [1984]. Don L. Anderson and Toshiro Tanimoto read the early stage of manuscript. Dave Scott and Caltech Japanese Mafia (Kiyoshi Yomogida and Hitoshi Kawakatsu) gave me useful comments. Contribution No. 4381 of the Division of Geological and Planetary Sciences, California Institute of Technology, Pasadena, California 91125.

\section{References}

Aki, K., Seismological evidence for the existence of soft thin layers in the upper mantle under Japan, $J$. Geophys. Res., 7s, 585-596, 1968.

Anderson, D. L., and A. M. Dziewonski, Upper mantle anisotropy: evidence from free oscillations, Geophys. J. R. astr. Soc., 69,,383-404,1982.

Anderson, D. L., and C. Sammis, Partial melting in 
the upper mantle, Phys. Earth Planet. Interiors, 3, 41-50, 1970.

Backus, G. E., Long-wave elastic anisotropy produced by horizontal layering, J. Geophys. Res., 67, 44274440, 1962.

Cathles, L. M., The Viscosity of the Earth's Mantle, Princeton Univ. Press, Princeton, N. J., 1975.

Chandrasekhar, S., Hydrodynamic and Hydromagnetic stability, Claredon Press, Oxford, 1961.

Christensen, U. Anisotropic viscosity, postglacial rebound, and mantle convection (abstract), Terra cognita, 6, 295, 1986.

Hager, B. H., and R. J. O'Connel, A simple global model of plate dynamics and mantle convection, $J$. Geophys. Res., 86, 4843-4867,1981.

Houston, M. H., and J. De Bremaecker, Numerical models of convection in the mantle, J. Geophys. Res., 80, 742-751, 1975.

Love, A. E., A Treaties on the Mathematical Theory of Elasticity, Cambridge University Press, 1926.
Richter, F. M., and S. F. Daly, Convection models having a multiplicity of large horizontal scales, $J$. Geophys. Res., 83, 4951-4956, 1978.

Saito, M., and Y. Abe, Consequences of anisotropic viscosity in the earth's mantle, Zisin, 37, 237-245, 1984 (in Japanese with English abstract)

Takeuchi, H., Y. Hamano, and Y. Hasegawa, Rayleigh- and Love-wave discrepancy and the existence of magma pockets in the upper mantle, J. Geophys. Res., 73, 3349-3350, 1968.

Waff, H. S., Effects of the gravitational field on liquid distribution in partial melts within the upper mantle, J. Geophys. Res., 85, 1815-1825, 1980.

S. Honda, Seismological Laboratory, 252-21, California Institute of Technology, Pasadena, CA 91125.

(Received September 2, 1986; accepted September 26, 1986.) 